Retrospective Study

\title{
Factors Associated with Successful Responses to Transforaminal Balloon Adhesiolysis for Chronic Lumbar Foraminal Stenosis: A Retrospective Study
}

Doo Hwan Kim, MD', Seong-Sik Cho, MD2,3, Yeon-Jin Moon, MD', Koo Kwon, MD', Kunhee Lee, MD', Jeong-Gil Leem, MD, PhD'1, Jin-Woo Shin, MD, PhD'1, Ji Hyun Park, MD4, and Seong-Soo Choi, MD, PhD ${ }^{1}$

From: ${ }^{1}$ Department of Anesthesiology and Pain Medicine, Asan Medical Center, University of Ulsan College of Medicine, Seoul, Republic of Korea; ${ }^{2}$ Department of Occupational and Environmental Health, Graduate School of Public Health, Seoul National University, Seoul, Gwanakgu, Republic of Korea; ${ }^{3}$ Department of Occupational and Environmental Medicine, Konkuk University Chungju Hospital, Chungju, Republic of Korea; ${ }^{4}$ Department of Anesthesiology and Pain Medicine, Hangang Sacred Heart Hospital, Hallym University College of Medicine, Seoul, Korea

Address Correspondence: Seong-Soo Choi, MD, PhD

Department of Anesthesiology and

Pain Medicine, Asan Medical Center, University of Ulsan College of Medicine 88 Olympic-ro 43 gil, Songpa-gu, Seoul 05505, Republic of Korea Email: choiss@amc.seoul.kr

Ji Hyun Park, MD

Department of Anesthesiology and Pain Medicine, Hangang Sacred Heart Hospital, College of Medicine, Hallym University,

12 Beodeunaru-ro 7 gil, Yeongdeungpogu, Seoul, 94200, Republic of Korea. Email: jihyunp8o@hanmail.net

Disclaimer: There was no external funding in the preparation of this manuscript.

Conflict of interest: Each author certifies that he or she, or a member of his or her immediate family, has no commercial association (i.e., consultancies, stock

ownership, equity interest, patent/ licensing arrangements, etc.) that might pose a conflict of interest in connection with the submitted manuscript.

Manuscript received: 11-08-2016 Revised manuscript received: o2-24-2017 Accepted for publication: 03-13-2017

Free full manuscript: www.painphysicianjournal.com
Background: Recently, transforaminal balloon adhesiolysis was introduced to manage patients with chronic radicular pain occurring with or without low back pain. However, the factors associated with successful responses to transforaminal balloon adhesiolysis are not known.

Objective: To evaluate the factors associated with successful responses to transforaminal balloon adhesiolysis for chronic lumbar spinal stenosis.

Study Design: This is a retrospective observational study.

Setting: A single pain clinic of tertiary medical center in Seoul, Republic of Korea.

Methods: From January 2013 to December 2014, a retrospective review of 199 patients with chronic lumbar foraminal stenosis, who were scheduled for transforaminal balloon adhesiolysis, was performed. Patients were considered successful responders if they showed either of the following: 1) a decrease of more than $50 \%$ on the numerical rating scale or 2) a decrease of more than $30 \%$ on the numerical rating scale and improved functional status 3 months after transforaminal balloon adhesiolysis. Logistic regression analysis was performed to determine the factors associated with successful responses to this surgical procedure.

Results: Three months after the transforaminal balloon adhesiolysis, $49.4 \%$ of patients were considered successful responders. Multivariate logistic regression analysis showed that factors other than degenerative disc herniation were independently associated with successful responses 3 months after this surgical procedure (odds ratio $=0.327 ; 95 \%$ confidence interval $=0.129-0.827 ; P=0.018$.

Limitations: The definition of successful response used in this study differed from the ones used in previous studies; a different definition may have led to different results. Further, the effects of other factors (ballooning, drugs, and saline washes) could not be excluded from our study. In addition, the correct method of assessing functional status, the Oswestry Disability Index, could not be used in this study; hence, the final results may have been affected.

Conclusion: These results suggest that transforaminal balloon adhesiolysis can successfully lead to improvement of symptoms in patients with chronic lumbar foraminal stenosis caused primarily by degenerative disc herniation.

Institutional Review Board (IRB) approval number: 2016-0228

Key words: Balloon, epidural adhesiolysis, chronic pain, radicular pain, lumbar spine, foraminal stenosis, degenerative disc

Pain Physician 2017; 20:E841-E848 
umbar spinal stenosis commonly leads to pain in the low back and lower extremities, impaired walking, and other forms of disability in the elderly. This disease is the most common indication for lumbar spinal surgery in patients older than 65 years of age. Although more than $80 \%$ of patients have partial symptomatic relief after surgery for spinal stenosis, 7 to 10 years later, at least one-third of patients develop low back pain (1). Post lumbar surgery syndrome, which is recurrent or persistent pain or sciatica after lumbar surgery, occurs in $10 \%-40 \%$ of patients (2). Therefore, non-surgical management, such as exercise, pharmacotherapy, and epidural steroid injection, often comprises first-line treatment unless an absolute indication for surgery is present. Lumbar epidural steroid injections are effective in managing chronic low back and lower extremity pain $(3,4)$; however, they are not always effective for leg pain and neurogenic intermittent claudication $(5,6)$. Percutaneous epidural adhesiolysis has been performed in many patients with chronic spinal stenosis who are unresponsive to conservative treatments, including epidural steroid injections (7). Recently, Kim et al found that transforaminal balloon adhesiolysis led to functional improvement and significant pain relief in patients with lumbar foraminal stenosis (4). Several studies also demonstrated the efficacy of balloon adhesiolysis in patients with chronic low back and leg pain (810). Although the effectiveness of transforaminal balloon adhesiolysis has been proven in prior studies, the factors associated with successful responses to transforaminal balloon adhesiolysis in patients with chronic lumbar spinal stenosis are not well known. To avoid unnecessary cost and inconvenience to the patient, transforaminal balloon adhesiolysis must be used only if the patient shows the proper indications for the treatment. Therefore, in this study, we aimed to identify the independent factors related to successful responses to transforaminal balloon adhesiolysis in patients with chronic lumbar spinal stenosis.

\section{Methods}

\section{Patients}

This retrospective study was performed at the pain clinic in our institution. This study protocol was approved by our institutional review board (approval number 2016-0228). We searched our institution's Information Technology of Service Management (ITSM) system between January 2014 and December 2015 with the terms "transforaminal epidural block with balloon," "chronic low back or leg pain," and "lumbar spinal stenosis." Patients were included in the study only if they met the following conditions: 1) they were at least 20 years of age; 2 ) they had chronic unilateral leg pain with or without low back pain for over 3 months; 3) they were diagnosed with lumbar spinal stenosis using a lumbar magnetic resonance image (MRI); and 4) symptoms were not relieved, or had not subsided within only one month with previous transforaminal or interlaminar epidural steroid injection combined with exercise, medical treatment, or physiotherapy. We excluded the patients who had any of the following conditions: 1) acute back or leg pain for less than 3 months; 2) axial low back pain; 3) allergy to local anesthetics, contrast dye, or steroids; 4) coagulopathy; 5) signs of progressive neurological deficits or motor weakness; 6) claudication due to vascular causes; 7) pregnancy or lactation; 8) systemic infection or injection site infection; 9) malignancy; and 10) an unstable medical or psychiatric condition.

\section{Procedure: Transforaminal Balloon Adhesiolysis}

All procedures were performed on an outpatient basis. A fluoroscope was used to visualize the needle and the catheter during the procedure. Medications or sedatives were not used prior to the procedure to prevent incidental neural damage and to allow cooperation by the patients during the procedure. The patient was placed on the table in a prone position with a pillow under the abdomen to minimize lumbar lordosis. After sterile preparation for the procedure, skin and soft tissue were anesthetized with $1 \%$ lidocaine. A 16-gauge R-K needle (Epimed International, Gloversville, NY) was inserted into the target intervertebral foramen associated with each patient's symptom. After obtaining a epidurogram using a contrast medium (Omnipaque, Nycomed Imaging, Oslo, Norway) prior to balloon insertion, a 3-French Fogarty catheter (Edward Lifescience, Irvine, CA) was then introduced into the epidural space of the target intervertebral foramen through the R-K needle and was advanced into the stenotic area (Fig. 1). The R-K needle was then partially withdrawn to prevent tearing the balloon catheter with the sharp edge of the bevel. The needle tip was held outside the foraminal inlet. Careful mechanical adhesiolysis was conducted by repeatedly inflating and deflating the balloon at the target region, which was at least 5 consecutive points from the lateral recess to the outlet of the neural fora- 


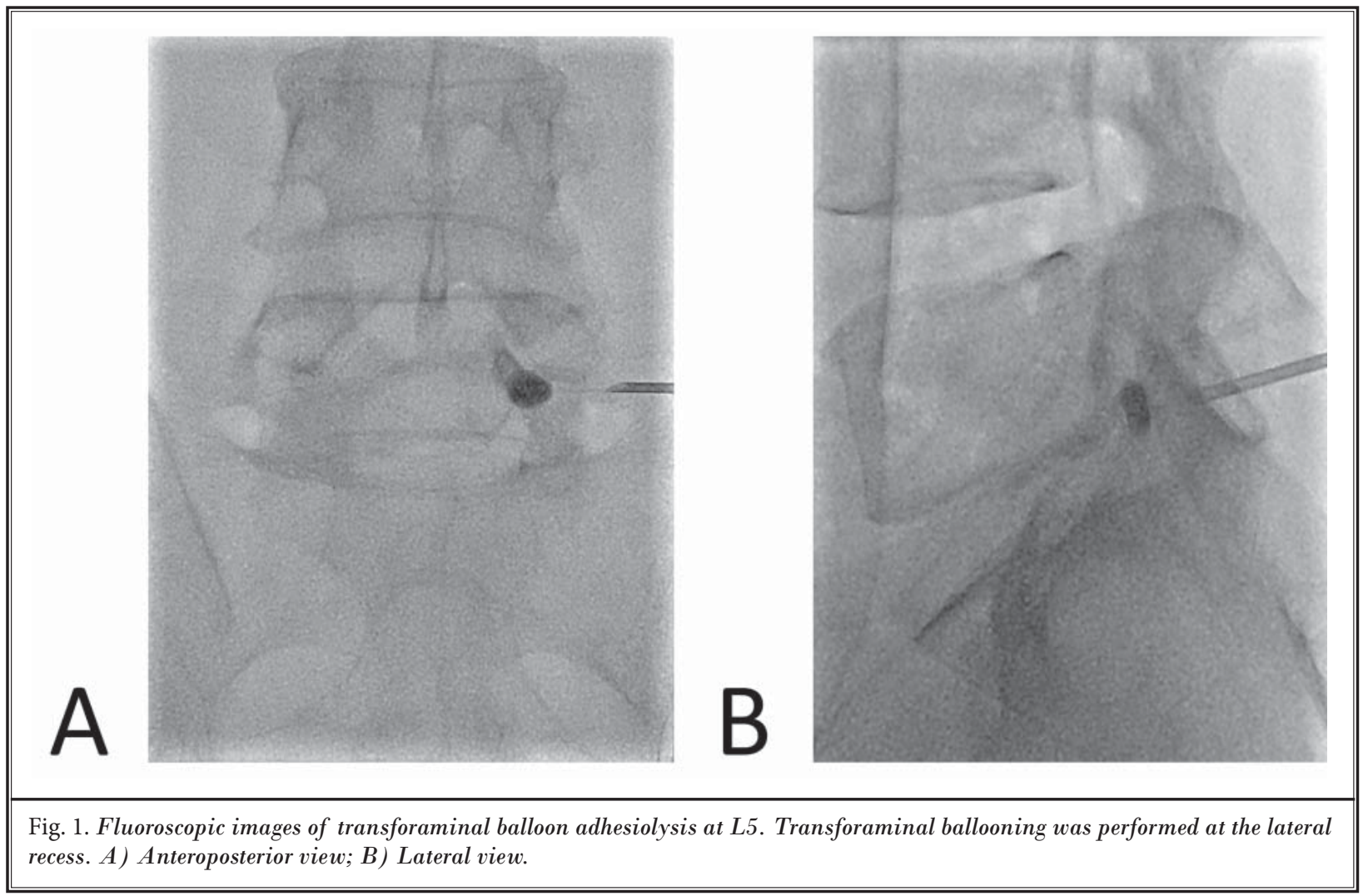

men. Each duration of balloon inflation was restricted to 5 seconds and repeated 3 times. The extent of balloon inflation was adjusted to the degree of pain; if moderate to severe pain was noticed during balloon inflation, further attempts were not made for safety reasons. The catheter moved only in the deflated state after adhesiolysis. The catheter had been prefilled with a contrast medium, and when $0.13 \mathrm{~mL}$ of the contrast medium was injected, the maximum diameter of the inflated balloon was determined within $6 \mathrm{~mm}$. After the transforaminal balloon adhesiolysis, the Fogarty catheter was carefully removed. The R-K needle was reinserted to administer an epidural steroid. After the injection of the contrast medium, the tip was confirmed to be in the anterior epidural space, and the epidurogram after balloon adhesiolysis was obtained to estimate the degree of improvement of filling defect. Then, $3 \mathrm{~mL}$ of a mixture of $1 \%$ lidocaine, $5 \mathrm{mg}$ of dexamethasone, and $1500 \mathrm{IU}$ of hyaluronidase was administered.

\section{Outcome Evaluation and Factors Associated with Successful Responses}

The outcome evaluation was performed at baseline and at one and 3 months after the procedure. For the outcome assessment, the numeric rating scale (NRS) and improvement in physical functional status were reviewed from each patient's medical record. Baseline characteristics such as age, gender, body mass index, underlying diseases, duration of pain, pain intensity, presence of spondylolisthesis, and previous operations were obtained for analysis. Foraminal stenosis grade and main stenotic pathology were analyzed with lumbar MRIs. MRIs was graded for lumbar foraminal stenosis in accordance with the following definitions (11): 1) Grade 0 , the absence of foraminal stenosis; 2 ) Grade 1, mild foraminal stenosis; 3) Grade 2, moderate foraminal stenosis; 4) Grade 3, severe foraminal stenosis. Lumbar foraminal stenosis can have various causes such as arthritic changes in the facet joints, disc herniation, osteophytes, and increased ligament flavum thickness. Although lumbar foraminal stenosis can be caused by various components, there were main pathologic components obviously (12). Disc herniation was more common among younger people (13), whereas the rates of facet osteoarthritis, osteophytes, and ligament flavum thickness increased with age, a finding that implies progressive degeneration (14-16). We reviewed the lumbar MRIs to determine the main stenotic pathology 
of spinal stenosis and divided our findings into 3 groups: 1) disc herniation; 2) causes other than disc herniation (facet osteoarthritis, osteophytes, and ligament flavum thickness); and 3) combined (disc herniation and other causes contributed equally) (Fig. 2). Patients were considered successful responders if they showed either of the following: 1) a decrease of $>50 \%$ on the numerical rating scale (NRS), or 2) a decrease of $>30 \%$ on the NRS and improved functional status 3 months after transforaminal balloon adhesiolysis $(9,17,18)$. Amelioration of walking distance and activities of daily living were regarded as improvements in physical functional status.

\section{Statistical Analysis}

The data were analyzed by using the Statistical Package for the Social Sciences Version 21.0 (SPSS Inc., Chicago, IL). Continuous demographic data from the non-responders and successful responders were compared by using the Student's t-test or the Mann-Whitney U-test and were documented as means with standard deviations or medians with interquartile ranges. Categorical demographic data were compared using a chi-square test or a Fisher's exact test. By using univariate and multivariate regression, the factors associated with successful responses 3 months after transforaminal balloon adhesiolysis were analyzed. The most relevant factors associated with successful responses were included in the univariate logistic regression analysis. The inclusion of variables in the final multivariate logistic regression analysis to evaluate independent factors associated with successful responses was based on biological plausibility, clinical importance, and statistical considerations. The goodness of fit of the model was assessed with the Hosmer-Lemeshow test. A value of $P$ $<0.05$ was considered statistically significant.

\section{Results}

As a result of searching ITSM, we found 199 patients who had been diagnosed with lumbar spinal stenosis and were scheduled for transforaminal balloon adhesiolysis. Of these patients, 25 did not appear or refused scheduled transforaminal balloon adhesiolysis, 84 underwent another procedure, 10 showed failed adhesiolysis because the balloon ruptured or the Fogarty catheter could not be advanced, and 3 underwent spine surgery without an epidural block. Finally, 77 patients who met all the inclusion criteria successfully underwent transforaminal balloon adhesiolysis. One month later, 20 patients had minimal reduction of pain or no improvement in functional status. Therefore, they were treated with other procedures such as epidural steroid blocks, percutaneous epidural neuroplasty, or pulsed radiofrequency ablation. After 3 months, 57 patients remained. Twenty-eight patients showed decreases on the NRS that were $>50 \%$. Ten patients presented improved functional status and decreases on the NRS that were $>30 \%$. Nineteen patients reported decreases that were $<30 \%$ on the NRS (Fig. 3). Therefore, 38 patients

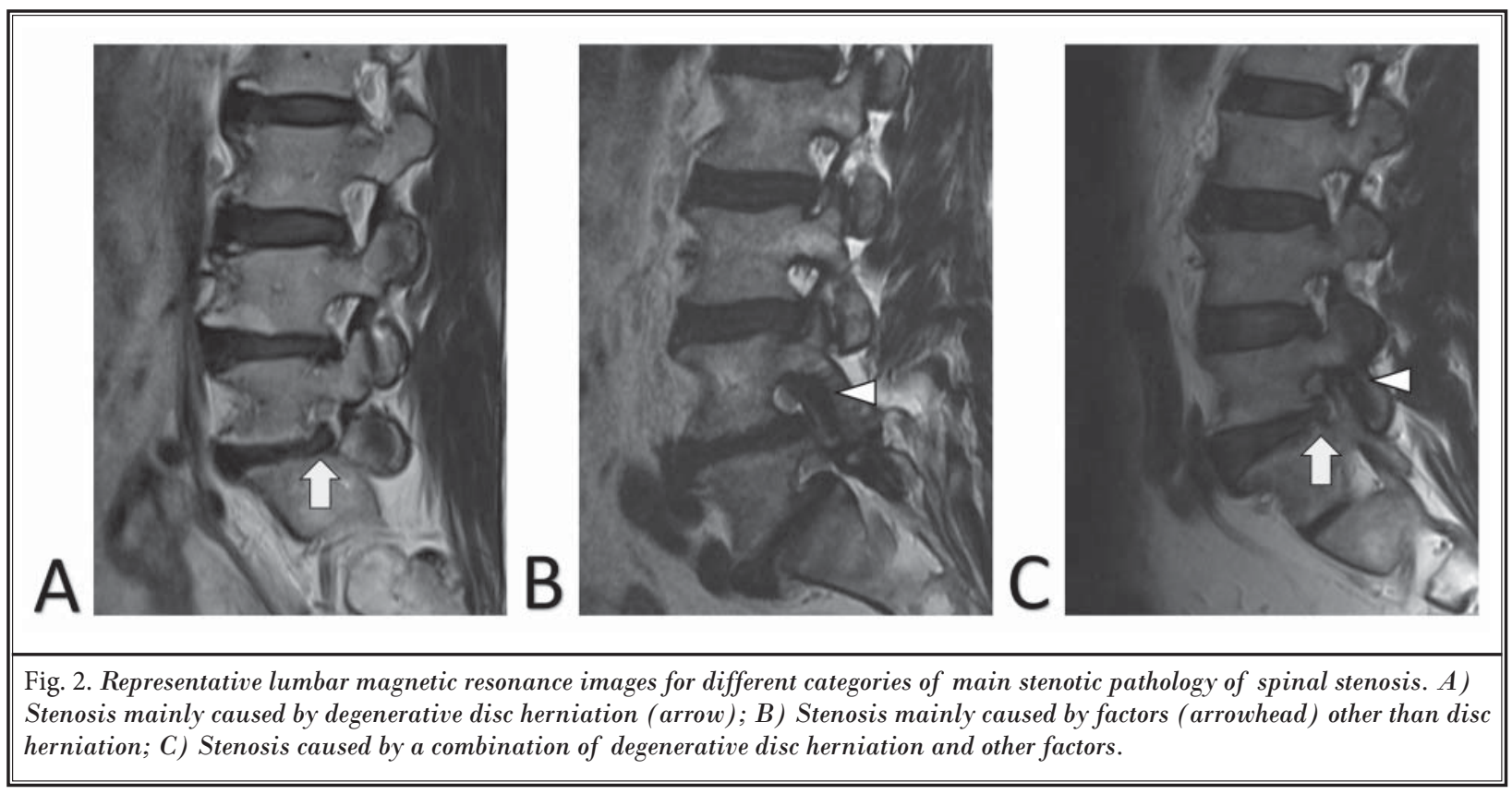


Factors Related to Successful Transforaminal Balloon Adhesiolysis

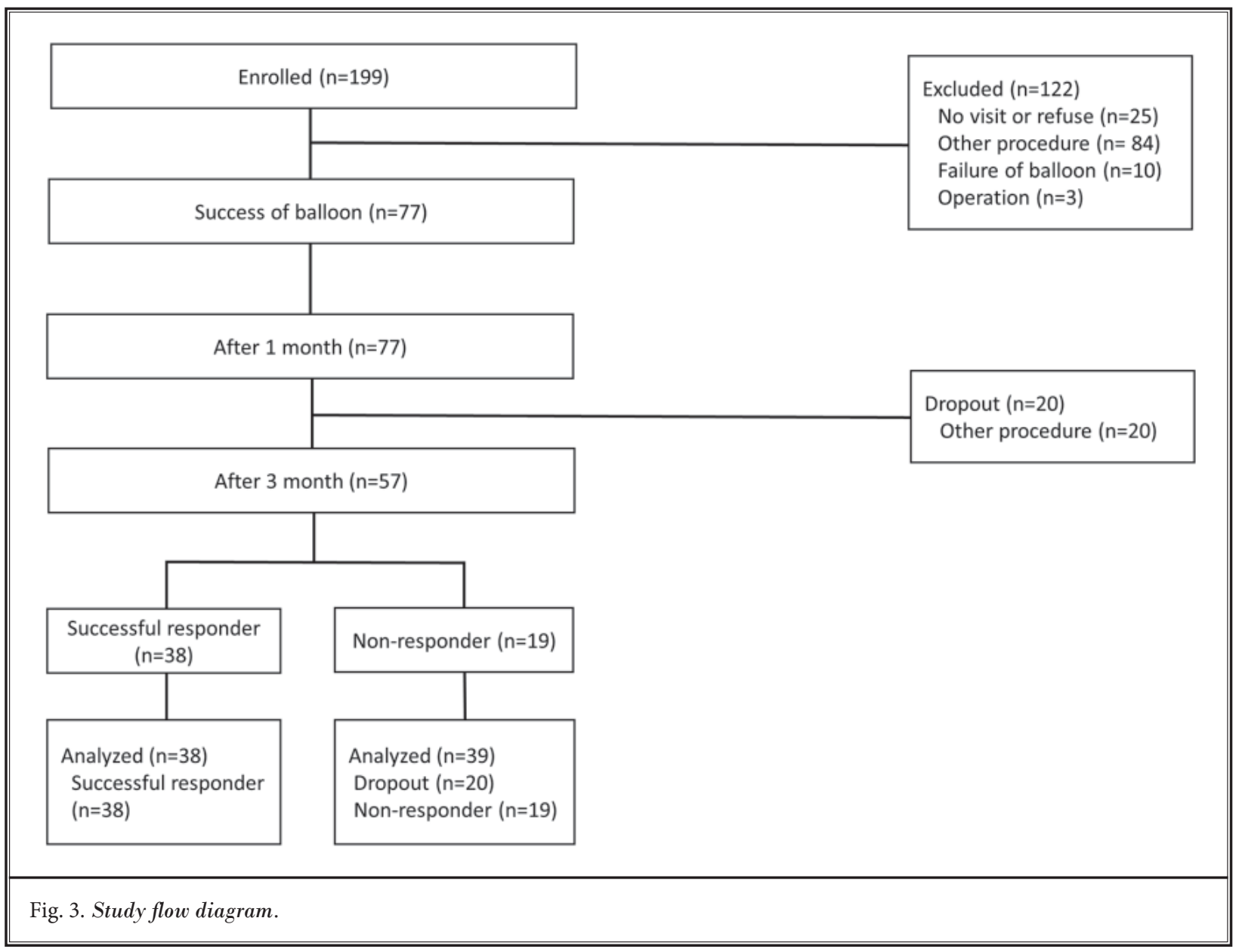

Table 1. Patient characteristics.

\begin{tabular}{|l|c|c|c|}
\hline & Non-responder $(\mathbf{n}=39)$ & Successful Responder (n = 38) & P value \\
\hline Age (years) & $65.3 \pm 11.1$ & $57.5 \pm 15.5$ & 0.014 \\
\hline Gender (male/female) & $22(56.4 \%) / 17(43.6 \%)$ & $19(50.0 \%) / 19(50.0 \%)$ & 0.573 \\
\hline BMI $\left(\mathrm{kg} / \mathrm{m}^{2}\right)$ & $23.9 \pm 2.1$ & $24.5 \pm 3.5$ & 0.487 \\
\hline DM (no/yes) & $39(100 \%) / 0(0 \%)$ & $36(94.7 \%) / 2(5.3 \%)$ & 0.240 \\
\hline Duration of pain (months) & $26.0(10.0-45.0)$ & $25.0(11.8-36.0)$ & 0.919 \\
\hline Pain intensity (NRS) & $7.0(6.0-8.0)$ & $6.5(6.0-8.0)$ & 0.979 \\
\hline Spondylolisthesis (no/yes) & $23(59.0 \%) / 16(41.0 \%)$ & $26(68.4 \%) / 12(31.6 \%)$ & 0.389 \\
\hline Previous operation (no/yes) & $35(89.7 \%) / 4(10.3 \%)$ & $34(89.5 \%) / 4(10.5 \%)$ & 1.000 \\
\hline $\begin{array}{l}\text { Stenosis grades } \\
\text { (mild/moderate/severe) }\end{array}$ & $20(51.3 \%) / 8(20.5 \%) / 11(28.2 \%)$ & $22(57.9 \%) / 7(18.4 \%) / 9(23.7 \%)$ & 0.840 \\
\hline Main stenotic pathology & & & 0.056 \\
\hline Disc herniation & $7(17.9 \%)$ & $13(34.2 \%)$ & $14(36.8 \%)$ \\
\hline Other than disc herniation & $25(64.1 \%)$ & $11(28.9 \%)$ & 0.696 \\
\hline Combined & $7(17.9 \%)$ & $9(7.4 \%) / 28(73.7 \%) / 1(2.6 \%)$ & \\
\hline Target level (L4/L5/S1) & $6(15.4 \%) / 32(82.1 \%) / 1(2.6 \%)$ & & \\
\hline
\end{tabular}

Data are expressed as mean \pm standard deviation $(\mathrm{SD})$, numbers (\%), or medians (interquartile range).

$\mathrm{BMI}=$ body mass index; $\mathrm{DM}=$ diabetes mellitus; $\mathrm{NRS}=$ numeric rating scale . 
Pain Physician: September/October 2017: 20: E841-E848

Table 2. Logistic regression analysis of factors associated with successful response at 3 months after transforaminal balloon adhesiolysis.

\begin{tabular}{|l|c|c|c|c|c|c||}
\hline & \multicolumn{3}{|c|}{ Univariate } & \multicolumn{3}{c|}{ Multivariate } \\
\hline Parameters & OR & $95 \%$ CI & $P$ value & OR & $95 \%$ CI & $P$ value \\
\hline Age & 0.956 & $0.920-0.993$ & 0.020 & 0.965 & $0.923-1.009$ & 0.115 \\
\hline Main stenotic pathology & & & & & & \\
\hline Disc herniation (reference) & 1.000 & & & 1.000 & & \\
\hline Other than disc herniation & 0.302 & $0.098-0.932$ & 0.037 & 0.327 & $0.129-0.827$ & 0.018 \\
\hline Combined & 0.846 & $0.226-3.167$ & 0.804 & 1.343 & $0.313-5.756$ & 0.691 \\
\hline
\end{tabular}

$\mathrm{OR}=$ odds ratio $\mathrm{CI}=$ confidence interval

$(49.4 \%)$ were considered successful responders in this study. Twenty patients who underwent another procedure after one month and 19 patients who reported decreases on the NRS that were $<30 \%$ were considered non-responders.

The demographic characteristics of non-responders and successful responders 3 months after transforaminal balloon adhesiolysis are summarized in Table 1. On average, successful responders were younger than nonresponders. A main stenotic pathology that comprised primarily disc herniation was more common among successful responders than among non-responders. Primary stenotic pathology comprising factors other than degenerative disc herniation was more common among non-responders than among successful responders. These findings had marginal statistical significance $(P$ $=0.056$ ). No significant differences in other baseline characteristics were seen between the 2 groups. Univariate logistic regression analysis showed that age and causes other than degenerative disc herniation were significantly associated with a successful response 3 months after transforaminal balloon adhesiolysis. However, after adjusting for demographic differences for multivariate regression analysis, the association between age and successful response was no longer significant. A stenotic pathology primarily comprising factors other than degenerative disc herniation was independently associated with successful responses 3 months after transforaminal balloon adhesiolysis (odds ratio $=0.327 ; 95 \%$ confidence interval $=0.129-0.827$; $P=0.018$ ) (Table 2).

\section{Discussion}

Various studies reported predictive factors (spondylolisthesis, previous lumbar spinal surgery, diabetes, and foraminal stenosis) that were associated with the effectiveness of percutaneous epidural adhesiolysis $(9,19,20)$. However, the factors associated with successful responses to transforaminal balloon adhesiolysis for chronic lumbar foraminal stenosis have not been evaluated. Multivariate logistic regression analysis in the present study showed that an independent factor associated with successful responses to transforaminal balloon adhesiolysis was degenerative disc herniation as a primary component of lumbar foraminal stenosis. Disc herniation, being the main stenotic cause, seemed to be the only reliable prognostic factor for transforaminal balloon adhesiolysis in the present study.

Lumbar foraminal stenosis is defined as the narrowing of the outlet of the nerve root caused by a decrease in the height of an intervertebral disc, facet arthritis, osteophytes, hypertrophy of the ligamentum flavum, or herniation of an intervertebral disc (11). A combination of several factors can cause the stenosis of neural foramina and the compression of nerve roots. Subsequently, continuous compression on the nerve roots and the surrounding venules causes inflammation, perineural edema, and ischemic injury (21). Degenerative disc herniation also induces perineural inflammation and ischemia through inflammatory mediators and mechanical compression. Both spinal stenosis and disc herniation lead to inflammation, venous outflow obstruction, perineural fibrosis, and finally, epidural adhesions (22). Therefore, inflammation and epidural adhesions may be more intense and occur more often in cases of lumbar spinal stenosis caused primarily by degenerative disc herniation than in cases with other primary causes. Epidural adhesiolysis showed positive clinical outcomes for the treatment of disc herniation with epidural adhesion because the injectate was spread well on the target region (23-27). Recently introduced balloon adhesiolysis can remove perineural adhesions and reduce mechanical irritation and venous congestion more effectively than conventional epidural adhesiolysis $(4,9)$. Moreover, balloon adhesiolysis can decompress the stenotic area so that the injectates can reach the target region. These injectate can wash inflammatory mediators from the stenotic region and 
ameliorate inflammation and inflammatory mediators by steroid. Therefore, balloon adhesiolysis reduces not only mechanical irritation but also chemical irritation caused by intradiscal inflammatory mediators effectively. If lumbar foraminal stenosis caused primarily by disc herniation is the main cause of inflammation, epidural adhesion, and fibrosis, then transforaminal balloon adhesiolysis might be able resolve these conditions effectively for all the reasons listed previously. Therefore, we speculated that transforaminal balloon adhesiolysis could be effective in lumbar foraminal stenosis caused primarily by disc herniation. Moreover, osteophytes or the hypertrophy of facet joints may lead to bony narrowing of the foramen inlet that may disturb engagement of the balloon catheter into the foramen. Among cases of facet hypertrophy, the success rate of accessing the ventral epidural space was lower with the transforaminal approach than with the modified interlaminar approach (28). This result suggests that transforaminal balloon adhesiolysis may be difficult to perform in cases of bone changes such as facet hypertrophy or osteophyte development. Consequently, transforaminal balloon adhesiolysis might be not effective in patients with principle stenotic causes other than disc herniation.

Young age seemed to be associated with successful responses to transforaminal balloon adhesiolysis in univariate regression analysis. However, after adjustment for main stenotic pathology in multivariate regression analysis, the association between age and successful responses could no longer be seen; a stenotic pathology primarily comprising disc herniation was the only independent factor associated with successful responses. Because lumbar disc herniation is common in young people (ages 30 - 50 years), young age might seem to be a predictor of successful responses to the procedure. Therefore, it is reasonably speculated that for younger patients, transforaminal balloon adhesiolysis can be more effective than conventional methods in treating radicular pain caused by disc herniation.
There were several limitations to this study. First, the definition of successful response used in this study slightly differed from the ones used in previous studies; a different definition may have led to different results. Second, the response to transforaminal balloon adhesiolysis seen at 3 months may be attributed to the combined effect of ballooning, the administration of various drugs, and flushing with saline. To exclude the drug effects, we chose the 3-month response as the target instead of the one-month response. However, the effects of local anesthetics, steroids, and saline washes are usually short-lived but can persist for over 3 months $(29,30)$. Thus, we did not exclude the effects of other factors on the successful response to transforaminal balloon adhesiolysis at 3 months. Third, improvements in walking distance and activities of daily living were considered ameliorations of functional status in our study. However, our indicators did not correctly represent physical functional status. The Oswestry Disability Index (ODI) is better method to measure physical functioning (31). However, we could not use the ODI because this was a retrospective study. Fourth, we evaluated the factors associated with successful responses to transforaminal balloon adhesiolysis until 3 months after the procedure. We could not search longterm effect such as 6 months or 12 months. Therefore, to overcome these limitations in the present study, we are currently conducting a randomized controlled trial to evaluate the effect of transforaminal balloon adhesiolysis on lumbar foraminal stenosis caused primarily by disc herniation.

\section{Conclusion}

Transforaminal balloon adhesiolysis can lead to significant pain relief and improvement in the physical functional statuses of patients with lumbar foraminal stenosis caused primarily by degenerative disc herniation. 


\section{References}

1. Katz JN, Harris MB. Clinical practice. Lumbar spinal stenosis. N Engl J Med 2008; 358:818-825.

2. Luft M. The failed back syndrome: Etiology and therapy. Orthopedics 1984; 7:946-948.

3. Manchikanti L, Buenaventura RM, Manchikanti KN, Ruan X, Gupta S, Smith HS, Christo PJ, Ward SP. Effectiveness of therapeutic lumbar transforaminal epidural steroid injections in managing lumbar spinal pain. Pain Physician 2012; 15:E199-E245.

4. Kim SH, Choi WJ, Suh JH, Jeon SR, Hwang CJ, Koh WU, Lee C, Leem JG, Lee SC, Shin JW. Effects of transforaminal balloon treatment in patients with lumbar foraminal stenosis: A randomized, controlled, double-blind trial. Pain Physician 2013; 16:213-224.

5. Armon C, Argoff CE, Samuels J, Backonja MM. Assessment: Use of epidural steroid injections to treat radicular lumbosacral pain: Report of the Therapeutics and Technology Assessment Subcommittee of the American Academy of Neurology. Neurology 2007; 68:723-729.

6. Fukusaki M, Kobayashi I, Hara T, Sumikawa K. Symptoms of spinal stenosis do not improve after epidural steroid injection. Clin J Pain 1998; 14:148-151.

7. Lee F, Jamison DE, Hurley RW, Cohen SP. Epidural lysis of adhesions. Korean J Pain 2014; 27:3-15.

8. Choi SS, Joo EY, Hwang BS, Lee JH, Lee G, Suh JH, Leem JG, Shin JW. A novel balloon-inflatable catheter for percutaneous epidural adhesiolysis and decompression. Korean J Pain 2014; 27:178-185.

9. Choi SS, Lee JH, Kim D, Kim HK, Lee S, Song KJ, Park JK, Shim JH. Effectiveness and factors associated with epidural decompression and adhesiolysis using a balloon-inflatable catheter in chronic lumbar spinal stenosis: 1-year follow-up. Pain Med 2016; 17:476-487.

10. Hwang B-Y, Ko H-S, Suh J-H, Shin J-W, Leem J-G, Lee J-D. Clinical experiences of performing transforaminal balloon adhesiolysis in patients with failed back surgery syndrome: Two cases report. Korean ] Anesthesiol 2014; 66:169-172.

11. Lee S, Lee JW, Yeom JS, Kim KJ, Kim HJ, Chung SK, Kang HS. A practical MRI grading system for lumbar foraminal stenosis. AJR Am J Roentgenol 2010; 194:1095-1098.

12. Lee CK, Rauschning W, Glenn W. Lateral lumbar spinal canal stenosis: Classifica- tion, pathologic anatomy and surgical decompression. Spine 1988; 13:313-320.

13. Jordan J, Konstantinou K, O'Dowd J. Herniated lumbar disc. BMJ Clin Evid 2011; 2011:1118.

14. Abbas J, Hamoud K, Masharawi YM, May $\mathrm{H}$, Hay O, Medlej B, Peled N, Hershkovitz I. Ligamentum flavum thickness in normal and stenotic lumbar spines. Spine 2010; 35:1225-1230.

15. Nathan H. Osteophytes of the vertebral column. J Bone Joint Surg Am 1962; 44:243-268

16. Schwarzer AC, Wang SC, Bogduk N, McNaught PJ, Laurent R. Prevalence and clinical features of lumbar zygapophysial joint pain: A study in an Australian population with chronic low back pain. Ann Rheum Dis 1995; 54:100-106.

17. Dworkin RH, Turk DC, Farrar JT, Haythornthwaite JA, Jensen MP, Katz NP, Kerns RD, Stucki G, Allen RR, Bellamy N, Carr DB, Chandler J, Cowan P, Dionne R, Galer BS, Hertz S, Jadad AR, Kramer LD, Manning DC, Martin S, McCormick CG, McDermott MP, McGrath P, Quessy S, Rappaport BA, Robbins W, Robinson JP, Rothman M, Royal MA, Simon L, Stauffer JW, Stein W, Tollett J, Wernicke J, Witter J; Immpact. Core outcome measures for chronic pain clinical trials: IMMPACT recommendations. Pain 2005; 113:9-19.

18. Geurts JW, van Wijk RM, Wynne HJ, Hammink E, Buskens E, Lousberg R, Knape JT, Groen GJ. Radiofrequency lesioning of dorsal root ganglia for chronic lumbosacral radicular pain: A randomised, double-blind, controlled trial. Lancet 2003; 361:21-26.

19. Lee JH, Lee SH. Clinical effectiveness of percutaneous adhesiolysis and predictive factors of treatment efficacy in patients with lumbosacral spinal stenosis. Pain Med 2013; 14:1497-1504.

20. Choi E, Nahm FS, Lee PB. Evaluation of prognostic predictors of percutaneous adhesiolysis using a Racz catheter for post lumbar surgery syndrome or spinal stenosis. Pain Physician 2013; 16:E531-E536.

21. Cooper RG, Freemont AJ, Hoyland JA, Jenkins JP, West CG, Illingworth KJ, Jayson MI. Herniated intervertebral discassociated periradicular fibrosis and vascular abnormalities occur without inflammatory cell infiltration. Spine 1995; 20:591-598.

22. Kobayashi S, Baba H, Uchida K, Kokubo
Y, Kubota C, Yamada S, Suzuki Y, Yoshizawa $\mathrm{H}$. Effect of mechanical compression on the lumbar nerve root: localization and changes of intraradicular inflammatory cytokines, nitric oxide, and cyclooxygenase. Spine 2005; 30:1699-1705.

23. Jamison DE, Hsu E, Cohen SP. Epidural adhesiolysis: An evidence-based review. J Neurosurg Sci 2014; 58:65-76.

24. Anderson SR, Racz GB, Heavner J. Evolution of epidural lysis of adhesions. Pain Physician 2000; 3:262-270.

25. Gerdesmeyer L, Wagenpfeil S, Birkenmaier C, Veihelmann A, Hauschild M, Wagner K, Muderis MA, Gollwitzer $H$, Diehl P, Toepfer A. Percutaneous epidural lysis of adhesions in chronic lumbar radicular pain: A randomized, double-blind, placebo-controlled trial. Pain Physician 2013; 16:185-196.

26. Gerdesmeyer L, Lampe R, Veihelmann A, Burgkart R, Gobel M, Gollwitzer H, Wagner K. Chronic radiculopathy: Use of minimally invasive percutaneous epidural neurolysis according to Racz. Schmerz 2005; 19:285-295.

27. Park EJ, Park SY, Lee SJ, Kim NS, Koh do Y. Clinical outcomes of epidural neuroplasty for cervical disc herniation. J Korean Med Sci 2013; 28:461-465.

28. Kim ED, Roh MS, Park JJ, Jo D. Comparison of the ventral epidural spreading in modified interlaminar approach and transforaminal approach: A randomized, double-blind study. Pain Med 2016; 17:1620-1627.

29. Lee JH, An JH, Lee SH. Comparison of the effectiveness of interlaminar and bilateral transforaminal epidural steroid injections in treatment of patients with lumbosacral disc herniation and spinal stenosis. Clin J Pain 2009; 25:206-210.

30. Manchikanti L, Cash KA, McManus CD, Damron KS, Pampati V, Falco FJ. Lumbar interlaminar epidural injections in central spinal stenosis: Preliminary results of a randomized, double-blind, active control trial. Pain Physician 2012; 15:51-63.

31. Chiarotto A, Maxwell LJ, Terwee CB, Wells GA, Tugwell P, Ostelo RW. RolandMorris Disability Questionnaire and Oswestry Disability Index: Which has better measurement properties for measuring physical functioning in nonspecific low back pain? Systematic review and metaanalysis. Phys Ther 2016; 96:1620-1637. 